\title{
Split General Mixed Variational Inequality Problem
}

\author{
Fengjiao Wang ${ }^{1, a}$, Yali Zhao ${ }^{2, b^{*}}$ \\ ${ }^{1}$ College of Mathematics and Physics , Bohai University, Jinzhon , Liaoning 121013,China \\ ${ }^{2}$ College of Mathematics and Physics, Bohai University, Jinzhon, Liaoning 121013, China \\ Email: wangjiao3075@163.com,Email: yalizhao2000@163.com
}

\begin{abstract}
Keywords: Split general mixed variational inequality problem; Split mixed variational inequality problem; Split general variational inequality problem; Iterative algorithms; Convergence analysis

Abstract. In this paper, we introduce a split general mixed variational inequality problem which is a natural extension of a split variational inequality problem, mixed variational and variational inequality problems in Hilbert spaces.Using the resolvent operator technique, we propose an iterative algorithm for a split general mixed variational inequality problem and discuss some special cases.Further,we discuss the convergence criteria of these iterative algorithms. The results presented in this paper generalize, unify and improve many previously known results for mixed variational and variational inequality problems.
\end{abstract}

\section{Introduction}

It is well known that the mixed variational inequality problem is a generalized form of a variational inequality problem, having applications in different areas of optimization, optimal control, operation research, economics equilibrium and free boundary value problems. The mixed variational inequality has been extensively studied including its various generalizations in a general setting. In recent years, considerable interest has been shown in developing various extensions and generalizations of split variational inequality problem. By using the projection method, the authors of [2] introduced and studied split general quasi-variational inequality problem. [1, 3] introduced gap function and global error bounds for generalized mixed quasivariational inequalities. [6] studied the split common null point problem. [8] introduced and studied algorithms for the split variational inequality problems. Kazmi and Rizvi [12] introduced the iterative approximation of a common solution of a split generalized equilibrium problem and a fixed point problem for nonexpansive semigroup. Some split variational inequality problems and some examples, see the references therein.

Inspired and motivated by the above work, in this paper, we introduce and study a split general mixed variational inequality problem (in short, SpGMVIP) which is a natural extension of a split variational inequality problem (in short SpVIP), mixed variational and variational inequality problems in Hilbert spaces. By using the resolvent operator technique, we propose an iterative algorithm for a split general mixed variational inequality problem and discuss some special cases. Furthermore, we discuss the convergence criteria of these iterative algorithms. It is of further research effort to extend the iterative method presented here to solving split variational inclusions [11] , the split equilibrium problem [12] and split general quasi-variational inequality problem[2].

Throughout the paper unless stated otherwise, for each $\mathrm{i} \in\{1,2\}$, let $\mathrm{H}_{\mathrm{i}}$ be a real Hilbert space with inner product $\langle\cdot$,$\rangle and norm \|\| \|_{\text {. Let }} f_{i}: H_{i} \rightarrow H_{i}, g_{i}: H_{i} \rightarrow H_{i}$ be nonlinear mappings and A: $H_{1} \rightarrow H_{2}$ a bounded linear operator with its adjoint operator $A^{*}$. Let $\varphi_{i}: H_{i} \rightarrow R \cup\{+\infty\}$ with $\operatorname{Im} g_{i} \cap \operatorname{dom} \partial \varphi_{i} \neq \varnothing, \partial \varphi_{i}$ denotes the subdifferential of a proper, convex and lower semi-continuous function $\varphi_{i}: H \rightarrow R \cup\{+\infty\}$.

An important generalization of the variational inequality is the mixed variational inequality problem. The mixed variational inequality problem (in short, MVIP) is to find $x_{1} \in H_{1}$ such that 


$$
\left\langle f_{1}\left(x_{1}\right), y_{1}-x_{1}\right\rangle+\varphi\left(x_{1}\right)-\varphi\left(y_{1}\right) \geq 0, \forall y_{1} \in H_{1} .
$$

In this paper, we consider the following split general mixed variational inequality problem (in short, SpGMVIP): Find $x_{1}^{*} \in H_{1}$ such that $g_{1}\left(x_{1}^{*}\right) \in \operatorname{dom} \partial \varphi_{1}$ and

$$
\left\langle f_{1}\left(x_{1}^{*}\right), x_{1}-g_{1}\left(x_{1}^{*}\right)\right\rangle+\varphi\left(g_{1}\left(x_{1}^{*}\right)\right)-\varphi\left(x_{1}\right) \geq 0, \quad \forall x_{1} \in H_{1},
$$

and such that $x_{2}^{*}=A x_{1}^{*}$ and $g_{2}\left(x_{2}^{*}\right) \in \operatorname{dom} \partial \varphi_{2}$ solves

$$
\left\langle f_{2}\left(x_{2}^{*}\right), x_{2}-g_{2}\left(x_{2}^{*}\right)\right\rangle+\varphi\left(g_{2}\left(x_{2}^{*}\right)\right)-\varphi\left(x_{2}\right) \geq 0, \quad \forall x_{2} \in H_{2} \text {. }
$$

Now, we observe a special case of SpGMVIP (2)-(3).

If we set $g_{i}=I_{i}$, where $I_{i}$ is an identity operator on $H_{i}$, then SpGMVIP (2)-(3) is reduced to the following split mixed variational inequality problem (in short, SpMVIP): Find $x_{1}^{*} \in H_{1}$ such that $x_{1}^{*} \in \operatorname{dom} \partial \varphi_{1}$ and

$$
\left\langle f_{1}\left(x_{1}^{*}\right), x_{1}-x_{1}^{*}\right\rangle+\varphi\left(x_{1}^{*}\right)-\varphi\left(x_{1}\right) \geq 0, \quad \forall x_{1} \in H_{1},
$$

and such that $x_{2}^{*}=A x_{1}^{*}$ and $x_{2}^{*} \in \operatorname{dom} \partial \varphi_{2}$ solves

$$
\left\langle f_{2}\left(x_{2}^{*}\right), x_{2}-x_{2}^{*}\right\rangle+\varphi\left(x_{2}^{*}\right)-\varphi\left(x_{2}\right) \geq 0, \quad \forall \mathrm{x}_{2} \in H_{2} \text {. }
$$

Which appears to be new.

If $\varphi_{i}: H \rightarrow R \cup\{+\infty\}$ is the indicator function of closed convex set $C_{i} \subset H_{i}$, then the split mixed variational inequality problem is reduced to split general variational inequality problem (in short, SpGVIP): Find $x_{1}^{*} \in C_{1}$ such that

$$
\left\langle f_{1}\left(x_{1}^{*}\right), x_{1}-g_{1}\left(x_{1}^{*}\right)\right\rangle \geq 0, \forall x_{1} \in C_{1},
$$

and such that $x_{2}^{*}=A x_{1}^{*} \in C_{2}$ solves

$$
\left\langle f_{2}\left(x_{2}^{*}\right), x_{2}-g_{2}\left(x_{2}^{*}\right)\right\rangle \geq 0, \quad \forall x_{2} \in C_{2} \text {. }
$$

If $\varphi_{i}: H \rightarrow R \cup\{+\infty\}$ is the indicator function of closed convex set $C_{i} \subset H_{i}$, and $g_{i}=I_{i}$, where $I_{i}$ is an identity operator on $H_{i}$, then the split mixed variational inequality problem is reduced to split variational inequality problem (in short, SpVIP): Find $x_{1}^{*} \in C_{1}$ such that

$$
\left\langle f_{1}\left(x_{1}^{*}\right), x_{1}-x_{1}^{*}\right\rangle \geq 0, \forall x_{1} \in C_{1},
$$

and such that $x_{2}^{*}=A x_{1}^{*} \in C_{2}$ solves $\left\langle f_{2}\left(x_{2}^{*}\right), x_{2}-g_{2}\left(x_{2}^{*}\right)\right\rangle \geq 0, \forall \mathrm{x}_{2} \in C_{2}$.

If we set $H_{1}=H_{2}, f_{1}=f_{2}, g_{i}=I_{i}$, then SpGMVIP (2)-(3) is reduced to MVIP (1).

\section{Iterative algorithms}

For each $i \in\{1,2\}$, it is well known that

$$
-f_{i}\left(x_{i}^{*}\right) \in \partial \varphi_{i}\left(g_{i}\left(x_{i}^{*}\right)\right) \text {. }
$$

Further, it is easy to see that the following is true:

$$
g_{i}\left(x_{i}^{*}\right)-\rho_{i} f_{i}\left(x_{i}^{*}\right) \in\left(I+\rho_{i} \partial \varphi_{i}\right) g_{i}\left(x_{i}^{*}\right) \text {. }
$$

We have

$$
g_{i}\left(x_{i}^{*}\right)=J_{\rho_{i}}^{\partial \varphi_{i}}\left(g_{i}\left(x_{i}^{*}\right)-\rho_{i} f_{1}\left(x_{1}^{*}\right)\right) \text {, }
$$

for $\rho_{i} \geq 0$, where $J_{\rho_{i}}^{\partial \varphi_{i}}: H_{i} \rightarrow H_{i}$ defined as $J_{\rho_{i}}^{\partial \varphi_{i}}(\cdot)=\left(I-\rho_{i} \partial \varphi\right)_{i}^{-1}(\cdot)$ is a resolvent operator of $\partial \varphi_{i}$. Based on the above arguments, we propose the following iterative algorithm for approximating a solution to SpGMVIP (2)-3). Let $\left\{\alpha^{n}\right\} \subseteq(0,1)$ be a sequence such that $\sum_{n=1}^{\infty} \alpha^{n}=+\infty$.

Algorithm 1. Given $x_{1}^{0} \in H_{1}$, compute the iterative sequences $\left\{x_{1}^{n}\right\}$ defined by the following of 
iterative schemes:

$$
\begin{gathered}
g_{1}\left(y^{n}\right)=J_{\rho}^{\partial \varphi_{1}}\left(g_{1}\left(x_{1}^{n}\right)-\rho_{1} f_{1}\left(x_{1}^{n}\right)\right), \\
g_{2}\left(z^{n}\right)=J_{\rho}^{\partial \varphi_{2}}\left(g_{2}\left(A y^{n}\right)-\rho_{2} f_{2}\left(A y^{n}\right)\right), \\
x_{1}^{n+1}=\left(1-\alpha^{n}\right) x_{1}^{n}+\alpha^{n}\left[y^{n}+\gamma A^{*}\left(z^{n}-A y^{n}\right)\right]
\end{gathered}
$$

For all $n=0,1,2, \cdots, \rho_{1}, \rho_{2}, \gamma>0$.

If $g_{i}=I_{i}$, then Algorithm 1 is reduced to the following iterative algorithm for SpMVIP (4)-(5):

Algorithm 2. Given $x_{1}^{0} \in H_{1}$, compute the iterative sequences $\left\{x_{1}^{n}\right\}$ defined by the following of iterative schemes:

$$
\begin{aligned}
& g_{1}\left(y^{n}\right)=J_{\rho}^{\partial \varphi_{1}}\left(x_{1}^{n}-\rho_{1} f_{1}\left(x_{1}^{n}\right)\right), \\
& g_{2}\left(z^{n}\right)=J_{\rho}^{\partial \varphi_{2}}\left(A y^{n}-\rho_{2} f_{2}\left(A y^{n}\right)\right), \\
& x_{1}^{n+1}=\left(1-\alpha^{n}\right) x_{1}^{n}+\alpha^{n}\left[y^{n}+\gamma A^{*}\left(z^{n}-A y^{n}\right)\right]
\end{aligned}
$$

For all $n=0,1,2, \cdots, \rho_{1}, \rho_{2}, \gamma>0$.

If $\varphi_{i}: H \rightarrow R \cup\{+\infty\}$ is the indicator function of closed convex set $C_{i} \subset H_{i}$, then Algorithm 1 is reduced to the following iterative algorithm for SpGVIP (6)-(7):

Algorithm 3([2]). Given $x_{1}^{0} \in C_{1}$, compute the iterative sequences $\left\{x_{1}^{n}\right\}$ defined by the following of iterative schemes:

$$
\begin{aligned}
& g_{1}\left(y^{n}\right)=P_{C_{1}}\left(g_{1}\left(x_{1}^{n}\right)-\rho_{1} f_{1}\left(x_{1}^{n}\right)\right), \\
& g_{2}\left(z^{n}\right)=P_{C_{2}}\left(g_{2}\left(A y^{n}\right)-\rho_{2} f_{2}\left(A y^{n}\right)\right), \\
& x_{1}^{n+1}=\left(1-\alpha^{n}\right) x_{1}^{n}+\alpha^{n}\left[y^{n}+\gamma A^{*}\left(z^{n}-A y^{n}\right)\right]
\end{aligned}
$$

For all $n=0,1,2, \cdots, \rho_{1}, \rho_{2}, \gamma>0$.

If $\varphi_{i}: H \rightarrow R \cup\{+\infty\}$ is the indicator function of closed convex set $C_{i} \subset H_{i}$, and $g_{i}=I_{i}$, where $I_{i}$ is an identity operator on $H_{i}$, then Iterative Algorithm 1 is reduced to the following iterative algorithm for SpVIP (8)-(9):

Algorithm 4([10]). Given $x_{1}^{0} \in C_{1}$, compute the iterative sequences $\left\{x_{1}^{n}\right\}$ defined by the following of iterative schemes:

$$
\begin{aligned}
& g_{1}\left(y^{n}\right)=P_{C_{1}}\left(x_{1}^{n}-\rho_{1} f_{1}\left(x_{1}^{n}\right)\right), \\
& g_{2}\left(z^{n}\right)=P_{C_{2}}\left(A y^{n}-\rho_{2} f_{2}\left(A y^{n}\right)\right), \\
& x_{1}^{n+1}=\left(1-\alpha^{n}\right) x_{1}^{n}+\alpha^{n}\left[y^{n}+\gamma A^{*}\left(z^{n}-A y^{n}\right)\right]
\end{aligned}
$$

For all $n=0,1,2, \cdots, \rho_{1}, \rho_{2}, \gamma>0$.

If we set $H_{1}=H_{2}, f_{1}=f_{2}, g_{i}=I_{i}$, for all $x_{i}, i \in\{1,2\}$, then Iterative Algorithm 1 is reduced to the following iterative algorithm for MVIP (1.1):

Algorithm 5. Given $x_{1}^{0} \in H_{1}$, compute the iterative sequences $\left\{x_{1}^{n}\right\}$ defined by the following of iterative schemes:

$$
\begin{aligned}
& y^{n}=J_{\rho}^{\partial \varphi_{1}}\left(x_{1}^{n}-\rho_{1} f_{1}\left(x_{1}^{n}\right)\right), \\
& x_{1}^{n+1}=\left(1-\alpha^{n}\right) x_{1}^{n}+\alpha^{n} y^{n} .
\end{aligned}
$$

Definition 1. A nonlinear mapping $f_{1}: H_{1} \rightarrow H_{1}$ is said to be (i) $\alpha_{1}$-strongly monotone if there exists a constant $\alpha_{1}>0$ such that $\left\langle f_{1}(x)-f_{1}(\bar{x}), x-\bar{x}\right\rangle \geq \alpha_{1}\|x-\bar{x}\|^{2}$ for all $x, \bar{x} \in H_{1}$, (ii) $\beta_{1}$-Lipschitz continuous if there exists a constant $\beta_{1}>0$ such that $\left\|f_{1}(x)-f_{1}(\bar{x})\right\| \leq \beta_{1}\left\|x_{1}-\bar{x}\right\| \quad$ for all $x, \bar{x} \in H_{1}$. 
Lemma1([9]). Let $\varphi: H \rightarrow R \cup\{+\infty\}$ be a proper convex lower semi-continuous function. Then for a constant $\rho>0$, the resolvent operator of its subdifferential mapping $J_{\rho}^{\partial \varphi}=(I-\rho \partial \varphi)^{-1}$ is nonexpansive, that is $\left\|J_{\rho}^{\partial \varphi}(x)-J_{\rho}^{\partial \varphi}(\bar{x})\right\| \leq\|x-\bar{x}\| \quad$ for all $x, \bar{x} \in H$.

\section{Main Results}

Theorem1. For each $i \in\{1,2\}$, let $g_{i}: H_{i} \rightarrow H_{i}$ be $\tau_{i}$-Lipschitz continuous such that $\left(g_{i}-I_{i}\right)$ is $\sigma_{i}$ -strongly monotone, where $I_{i}$ is the identity operator on $H_{i}$. Let $f_{i}: H_{i} \rightarrow H_{i}$ be $\alpha_{i}$-strongly monotone with respect to $g_{i}$ and $\beta_{i}$-Lipschitz continuous. Let $A: H_{1} \rightarrow H_{2}$ be a bounded linear operator and let $A^{*}$ be its adjoint operator. Suppose $x_{1}^{*} \in H_{1}$ is a solution to SpGMVIP (2)-(3). Then the sequence $\left\{x_{1}^{n}\right\}$ generated by Algorithm1 converges strongly to $x_{1}^{*}$ provided that the constant $\rho_{i}, \gamma$ satisfy the following conditions:

$\left|\rho_{1}-\frac{\alpha_{1}}{\beta_{1}^{2}}\right|<\frac{\sqrt{\alpha_{1}^{2}-\beta_{1}^{2}\left(1-k_{1}^{2}\right)}}{\beta_{1}^{2}}, \alpha_{1}>\beta_{1} \sqrt{\tau_{1}^{2}-k_{1}^{2}}, k_{1}=\frac{\sqrt{2 \sigma_{1}+1}}{1+2 \theta_{2}}, k_{1}<\tau_{1}$,

$\theta_{2}^{2}=\frac{\tau_{2}^{2}-2 \rho_{2} \alpha_{2}+\rho_{2}^{2} \beta_{2}^{2}}{2 \sigma_{2}+1}, \rho_{2}>0, \gamma \in\left(0, \frac{2}{\|A\|^{2}}\right)$.

Proof. Since $x_{1}^{*} \in H_{1}$ is a solution to SpGMVIP (2)-(3), then we hav

$g_{1}\left(x_{1}^{*}\right)=J_{\rho_{1}}^{\partial \varphi_{1}}\left(g_{1}\left(x_{1}^{*}\right)-\rho_{1} f_{1}\left(x_{1}^{*}\right)\right)$,

$g_{1}\left(A x_{1}^{*}\right)=J_{\rho_{2}}^{\partial \varphi_{2}}\left(g_{2}\left(A x_{1}^{*}\right)-\rho_{2} f_{2}\left(A x_{1}^{*}\right)\right)$.

For $\rho_{i}>0$. From Algorithm $1(12),(15)$ and Lemma 1, we have

$$
\begin{aligned}
\left\|g_{1}\left(y^{n}\right)-g_{1}\left(x_{1}^{*}\right)\right\| & =\left\|J_{\rho_{1}}^{\partial \varphi_{1}}\left(g_{1}\left(x_{1}^{n}\right)-\rho_{1} f_{1}\left(x_{1}^{n}\right)\right)-J_{\rho_{1}}^{\partial \varphi_{1}}\left(g_{1}\left(x_{1}^{*}\right)-\rho_{1} f_{1}\left(x_{1}^{*}\right)\right)\right\| \\
& \leq\left\|g_{1}\left(x_{1}^{n}\right)-g_{1}\left(x_{1}^{*}\right)-\rho_{1}\left(f_{1}\left(x_{1}^{n}\right)-f_{1}\left(x_{1}^{*}\right)\right)\right\| .
\end{aligned}
$$

Next, since $f_{1}$ is $\alpha_{1}$-strongly monotone with respect to $g_{1}$ and $\beta_{1}$-Lipschitz continuous, and $g_{1}$ be $\tau_{1}$-Lipschitz continuous, we have

$$
\begin{aligned}
& \left\|g_{1}\left(x_{1}^{n}\right)-g_{1}\left(x_{1}^{*}\right)-\rho_{1}\left(f_{1}\left(x_{1}^{n}\right)-f_{1}\left(x_{1}^{*}\right)\right)\right\|^{2} \\
& =\left\|g_{1}\left(x_{1}^{n}\right)-g_{1}\left(x_{1}^{*}\right)\right\|^{2}-2 \rho_{1}\left\langle f_{1}\left(x_{1}^{n}\right)-f_{1}\left(x_{1}^{*}\right), g_{1}\left(x_{1}^{n}\right)-g_{1}\left(x_{1}^{*}\right)\right\rangle+\rho_{1}^{2}\left\|f_{1}\left(x_{1}^{n}\right)-f_{1}\left(x_{1}^{*}\right)\right\|^{2} \\
& \leq\left(\tau_{1}^{2}-2 \rho_{1} \alpha_{1}+\rho_{1}^{2} \beta_{1}^{2}\right)\left\|x_{1}^{n}-x_{1}^{*}\right\|^{2} .
\end{aligned}
$$

As a result we obtain

$$
\left\|g_{1}\left(y^{n}\right)-g_{1}\left(x_{1}^{*}\right)\right\| \leq \sqrt{\tau_{1}^{2}-2 \rho_{1} \alpha_{1}+\beta_{1}^{2}}\left\|x_{1}^{n}-x_{1}^{*}\right\| \text {. }
$$

Since $\left(g_{1}-I_{1}\right)$ is $\sigma_{1}$-strongly monotone, we have

$$
\begin{aligned}
\left\|y^{n}-x_{1}^{*}\right\|^{2} & \leq\left\|g_{1}\left(y^{n}\right)-g_{1}\left(x_{1}^{*}\right)\right\|-2\left\langle\left(g_{1}-I_{1}\right) y^{n}-\left(g_{1}-I_{1}\right) x_{1}^{*}, y^{n}-x_{1}^{*}\right\rangle \\
& \leq\left\|g_{1}\left(y^{n}\right)-g_{1}\left(x_{1}^{*}\right)\right\|^{2}-2 \sigma_{1}\left\|y^{n}-x_{1}^{*}\right\|^{2} .
\end{aligned}
$$

Which implies

$$
\left\|y^{n}-x_{1}^{*}\right\| \leq \frac{1}{\sqrt{2 \sigma_{1}+1}}\left\|g_{1}\left(y^{n}\right)-g_{1}\left(x_{1}^{*}\right)\right\| \text {. }
$$

From (17) and (18), we have

$\left\|y^{n}-x_{1}^{*}\right\| \leq \theta_{1}\left\|x_{1}^{n}-x_{1}^{*}\right\|$, 
where $\theta_{1}=\sqrt{\frac{\tau_{1}^{2}-2 \rho_{1} \alpha_{1}+\rho_{1}^{2} \beta_{1}^{2}}{2 \sigma_{1}+1}}$. Similarly, from Algorithm 1 (13), (16), Lemma 1 and using the facts that $f_{2}$ is $\alpha_{2}$-strongly monotone with respect to $g_{2}$ and $\beta_{2}$-Lipschitz continuous, $\left(g_{1}-I_{1}\right)$ is $\sigma_{1}$ -strongly monotone, and $g_{2}$ is $\tau_{2}$-Lipschitz continuous, we have

$$
\begin{aligned}
& \left\|g_{1}\left(z^{n}\right)-g_{1}\left(A x_{1}^{*}\right)\right\| \leq \sqrt{\tau_{2}^{2}-2 \rho_{2} \alpha_{2}+\rho_{2}^{2} \beta_{2}^{2}}\left\|A y^{n}-A x_{1}^{*}\right\| . \\
& \left\|z^{n}-A x_{1}^{*}\right\| \leq \theta_{2}\left\|A y^{n}-A x_{1}^{*}\right\|,
\end{aligned}
$$

where $\theta_{2}=\sqrt{\frac{\tau_{2}^{2}-2 \rho_{2} \alpha_{2}+\rho_{2}^{2} \beta_{2}^{2}}{2 \sigma_{2}+1}}$. Next, from Algorithm 1 (14), we have

$$
\left.\left\|x_{1}^{n+1}-x_{1}^{*}\right\| \leq\left(1-\alpha^{n}\right) \mid x_{1}^{n}-x_{1}^{*}\left\|+\alpha^{n}\right\| y^{n}-x_{1}^{*}-\gamma A^{*}\left(A y^{n}-A x_{1}^{*}\right)\|+\gamma\| A^{*}\left(z^{n}-A x_{1}^{*}\right) \|\right\}
$$

Further, using the definition of $A^{*}$, the fact that $A^{*}$ is a bounded linear operator with $\left\|A^{*}\right\|=\|A\|$, and given condition on $\gamma$, we have

$$
\begin{aligned}
& \left\|y^{n}-x_{1}^{*}-\gamma A^{*}\left(A y^{n}-A x_{1}^{*}\right)\right\|^{2} \\
& =\left\|y^{n}-x_{1}^{*}\right\|^{2}-2 \gamma\left\langle y^{n}-x_{1}^{*}, A^{*}\left(A y^{n}-A x_{1}^{*}\right)\right\rangle+\gamma^{2}\left\|A^{*}\left(A y^{n}-A x_{1}^{*}\right)\right\|^{2} \\
& \leq\left\|y^{n}-x_{1}^{*}\right\|^{2}-\gamma\left(2-\gamma\|A\|^{2}\right)\left\|A y^{n}-A x_{1}^{*}\right\|^{2} \\
& \leq\left\|y^{n}-x_{1}^{*}\right\|^{2} .
\end{aligned}
$$

From (21), we have

$$
\left\|A^{*}\left(z^{n}-A x_{1}^{*}\right)\right\| \leq\|A\|\left\|z^{n}-A x_{1}^{*}\right\| \leq \theta_{2}\|A\|\left\|A y^{n}-A x_{1}^{*}\right\| \leq \theta_{2}\|A\|^{2}\left\|y^{n}-x_{1}^{*}\right\| \text {. }
$$

Combining (23) and (24) with inequality (3.8), we have $\left\|x_{1}^{n+1}-x_{1}^{*}\right\| \leq\left[1-\alpha^{n}(1-\theta)\right] \mid x_{1}^{n}-x_{1}^{*} \|$, where $\theta=\theta_{1}\left(1+\gamma\|A\|^{2} \theta_{2}\right)$. Hence, after $n$ iterations, we obtain

$$
\left\|x_{n+1}-x^{*}\right\| \leq \prod_{j=1}^{n}\left[1-\alpha_{j}(1-\theta)\right] \mid x_{0}-x^{*} \| .
$$

It follows from the conditions on $\rho_{1}$ and $\rho_{2}$ that $\theta \in(0,1)$. Since $\sum_{n=1}^{\infty} \alpha^{n}=+\infty$ and $\theta \in(0,1)$, this implies in the light of [13] that $\lim _{n \rightarrow \infty} \prod_{j=1}^{n}\left[1-\alpha_{j}(1-\theta)\right]=0$.

Thus it follows from (3.11) that $\left\{x_{n}\right\}$ converges strongly to $x^{*}$ as $n \rightarrow+\infty$. Since A is continuous,it follows from (17) and (19)-(21) that $y^{n} \rightarrow x_{1}^{*}, g_{1}\left(y^{n}\right) \rightarrow g_{1}\left(x_{1}^{*}\right), A y^{n} \rightarrow A x_{1}^{*}$, $z^{n} \rightarrow A x_{1}^{*}$ and $g_{2}\left(z^{n}\right) \rightarrow g_{2}\left(A x_{1}^{*}\right)$ as $n \rightarrow+\infty$. This completes the proof.

If we set $g_{i}=I_{i}$, then Theorem 1 reduces to the following result for the convergence of Algorithm 2 for SpMVIP (4) -(5).

Corollary 2. For each $i \in\{1,2\}$, let $f_{i}: H_{i} \rightarrow H_{i}$ be $\alpha_{i}$-strongly monotone and $\beta_{i}$-Lipschitz continuous. Let $A: H_{1} \rightarrow H_{2}$ be a bounded linear operator and let $A^{*}$ be its adjoint operator. Suppose $x_{1}^{*} \in H_{1}$ is a solution to SpMVIP (4)-(5). Then the sequence $\left\{x_{1}^{n}\right\}$ generated by Algorithm 2 converges strongly to $x_{1}^{*}$ provided that the constants $\rho_{i}$ and $\gamma$ satisfy the following conditions:

$$
\left|\rho_{1}-\frac{\alpha_{1}}{\beta_{1}^{2}}\right|<\frac{\sqrt{\alpha_{1}^{2}-\beta_{1}^{2}\left(1-k_{1}^{2}\right)}}{\beta_{1}^{2}}, \alpha_{1}>\beta_{1} \sqrt{1-k_{1}^{2}}, k_{1}=\frac{1}{1+2 \theta_{2}},
$$




$$
\theta_{2}^{2}=1-2 \rho_{2} \alpha_{2}+\rho_{2}^{2} \beta_{2}^{2}, \rho_{2}>0, \gamma \in\left(0, \frac{2}{\|A\|^{2}}\right)
$$

If we set $H_{1}=H_{2}, f_{1}=f_{2}, g_{i}=I_{i}$, for all $x_{i}, i \in\{1,2\}$, then Theorem 1 is reduced to the following result for the convergence of Algorithm 5 for MVIP (1).

Corollary 3. For each $i \in\{1,2\}$, let $f_{1}: H_{1} \rightarrow H_{1}$ be $\alpha_{1}$-strongly monotone and $\beta_{1}$-Lipschitz continuous. Suppose $x_{1}^{*} \in H_{1}$ is a solution to MVIP (1). Then the sequence $\left\{x_{1}^{n}\right\}$ generated by Algorithm 5 converges strongly to $x_{1}^{*}$ provided that the constants $\rho_{1}$ satisfies $0<\rho_{1}<\frac{2 \alpha_{1}}{\beta_{1}^{2}}$.

\section{Acknowledgements.}

This work is supported by the National Natural Science Foundation of China (11371070).

\section{References}

[1] S. A. Khan and J.w.Chen. Gap function and global error bounds for generalized mixed quasi variational inequalities[J]. Applied Mathematics and Computation. 2015(260)71-81.

[2]K.R.Kazmi.Split general quasi-variational inequality problem[J]. Georgian Math. 2015(22)385-392.

[3] K.R. Kazmi and S.H. Rizvi. An iterative algorithm for generalized mixed equilibrium problem[J]. Afrika Matematika. 2014(25)857-867.

[4] A. Bensoussan and J.L.lions. Applications of variational inequalites in Stochastic Control, Stud. Math. North-Holland, Amsterdam. 1982(12).

[5] Zeng L C. Iterative algorithms for finding approximate solutions for general strongly nonlinear variational inequalities [J]. J. Math.Anal.Appl. 1994(187)352-360.

[6] C. Byren,Y. Censor, A. Gibali and S. Reich. Split common null point problem[J]. J. Nonliear Convex Anal.2012(13)759-775.

[7] R. Glowinski. Numerical methods for nonlinear problems. Springer, New York, (1984).

[8] Y. Censor and A. Gibali and S. Reich. Algorithms for the split variational inequality[J]. Numer. Algorithms. 2012(59)301-323.

[9] G. Minty. Monotone nonlinear operators in Hilbert space. Duke Math. J. 1962(29)341-346.

[10]K.R. Kazmi. Split nonconvex variational inequality problem. Math. Sci. (Springer) 2013(7 ).

[11]A. Moudafi. Split monotone variational inclusions[J]. J. Optim. Theory Appl.2011(150) 275-283.

[12] K.R. Kazmi and S.H. Rizvi. Iterative approximation of a common solution of a split generalized equilibrium problem and a fixed point problem for nonexpansive semigroup. Math. Sci.(Springer) 2013(7). 